\title{
Heat conduction across molecular junctions between nanoparticles
}

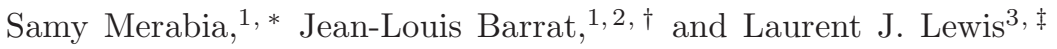 \\ ${ }^{1}$ LPMCN, Université de Lyon; UMR 5586 Université Lyon 1 et CNRS, F-69622 Villeurbanne, France \\ ${ }^{2}$ LIPHY, Université Grenoble 1 and CNRS, UMR 5588, Saint Martin d'Hères, F-38402, France \\ ${ }^{3}$ Département de Physique et Regroupement Québécois sur les Matériaux de Pointe (RQMP), \\ Université de Montréal, C.P. 6128, Succursale Centre-Ville, Montréal, Québec, Canada H3C $3 J 7$
}

(Dated: June 15, 2018)

\begin{abstract}
We investigate the problem of heat conduction across a molecular junction connecting two nanoparticles, both in vacuum and in a liquid environment, using classical molecular dynamics simulations. In vacuum, the well-known result of a length independent conductance is recovered; its precise value, however, is found to depend sensitively on the overlap between the vibrational spectrum of the junction and the density of states of the nanoparticles that act as thermal contacts. In a liquid environment, the conductance is constant up to a crossover length, above which a standard Fourier regime is recovered.
\end{abstract}

PACS numbers: 05.70.Ln

\section{INTRODUCTION}

Since the seminal work of Fermi, Pasta and Ulam [1], the problem of heat transfer in low dimensional systems has attracted much attention (see, e.g., Refs. 2, 33] for a review), in particular with regards to nanoscale systems. While the problem is fundamentally important and interesting, understanding heat transport in nanoscale systems (including molecular junctions) is of considerable importance for applications, e.g., in nano-electronic as well as thermo-electric devices where, typically, a minimal thermal conductivity is required to convert heat in electric current; molecular junctions are also promising systems for use as thermal rectifiers [4, 5].

The problem has been the object of numerous theoretical studies, which may be classified in two categories: (i) simple, toy-model one-dimensional systems - typically a chain - coupled to heat baths 6 - 8 ; ; (ii) numerical simulations of realistic systems. The latter category includes, among others, molecular dynamics studies of carbon nanotubes 9], self-assembled monolayers [10], molecular junctions [11] and polyethylene chains as well [12], which have been motivated to a large extent by experimental studies of a number of materials, including nanowires [13, 14], carbon nanotubes 15 17] and diamond nano-rods [18].

From a fundamental viewpoint, nanoscale heat transport is closely related to the study of the validity of Fourier's law at small spatial scales. In the context of a junction of length $L$ subject to a temperature difference $\Delta T$, the heat current $J$ is given by

$$
J=\kappa \frac{\Delta T}{L},
$$

where $\kappa$ is the thermal conductivity, which in general is a material property. In short junctions, the physical dimensions of the systems of interest is comparable to the phonon mean free path $\Gamma$, and one expects a transition from a ballistic regime, where $\kappa \sim L$ when $L<\Gamma$, to a diffusive regime when $L$ is large and temperature is high; in this case, $\kappa \sim L^{\alpha}$ where $0 \leq \alpha<1$, which may eventually lead to the divergence of the conductivity with system size for systems with vanishing concentrations of impurities.

The scaling of the thermal conductivity with length is often analysed using the Landauer formula [19] commonly used in mesoscopic physics:

$$
J=\int_{0}^{\infty} \frac{\bar{h} \omega}{2 \pi} \mathcal{T}(\omega)\left(f_{L}-f_{R}\right) d \omega
$$

where $\mathcal{T}(\omega)$ is the transmission coefficient of phonons, and $f_{L}$ and $f_{R}$ are the distributions of phonons in the two heat baths ('left' and 'right') to which the system is connecting. These distributions depend on the densities of vibrational states and on the thermal (Bose Einstein or classical) statistics In the limit where the temperature difference $\Delta T$ between the two heat baths vanishes and if the two baths have identical statistics, the Landauer formula becomes consistent with the Fourier law:

$$
J=\left(\int_{0}^{\infty} \frac{\bar{h} \omega}{2 \pi} \mathcal{T}(\omega) \frac{\partial f}{\partial T} d \omega\right) \Delta T
$$

with a conductivity increasing linearly with length: $\kappa \sim$ $L$.

The dependence of the thermal conductivity on the system length has been extensively studied in nanowires and carbon nanotubes [3]. There has been comparatively fewer studies in molecular junctions, largely because measuring heat currents across molecular junctions is extremely difficult. The usual way to measure heat current across a $1 \mathrm{D}$ object consists in connecting the object to two electrodes and measuring the heating Joule effect due to a difference of voltage between the electrodes. However, this gives access to the thermal conductance and one therefore needs a model for the nanoscale junction to extract the conductivity. Also, the thermal contacts 
of the junction may contribute to the Joule effect, thus making the determination of the conductance inaccurate. A promising but less explored method consists in using nanoparticles (NPs) as thermal contacts. Indeed, NPs of identical or different materials can be permanently linked with a few nanometers long molecule - e.g. an alcenes or complementary DNA strands. Such particle may then serve as thermal contacts, either to be inserted within a NEMS device for thermal transport, or manipulated, heated and thermally monitored using optical methods.

In the present article, we propose a numerical protocol [20, 21] to measure the thermal conductivity of a molecular junction permanently linking two NPs, possibly immersed in a liquid. We examine the steady state current/temperature difference for the junction, and we compare the conductivity in the linear regime for junctions in vacuum and in a liquid. We unveil the crucial role of the liquid solvent on the thermal conductivity of the junction with respect to its length. For a junction in vacuum, i.e., "dry", the thermal conductivity is found to increase linearly with the junction length $L$, in agreement with the Landauer formula, eq3. For a junction immersed in a liquid solvent, or "wet", in contrast, we find a different behaviour: when the junction is short, the conductivity increases approximately linearly with $L$, as for the dry case, then saturates for longer junctions; the difference results from the "friction" of the solvent, which damps the low-frequency modes of the junction.

\section{COMPUTATIONAL DETAILS}

We have used molecular dynamics (MD) simulations to model a molecular junction between two NPs, embedded or not in a liquid matrix, as illustrated in Fig. 1 The NPs each contain 555 atoms cut from an FCC lattice, interacting via the combination of a simple Lennard-Jones interaction and a nonlinear spring that forms the so called FENE interaction 22]. The stiffness and maximum extension of the nonlinear spring are $k_{\mathrm{FENE}}=30 \epsilon / \sigma$ and $l_{\max }=1.5 \sigma$, with $\sigma$ and $\epsilon$ the diameter and energy of the Lennard Jones potential (see below). The molecular junction, whose setup is displayed in Fig. 2, consists of $N$ beads $(N=5-25)$; each bead interacts with its neighbours via a Hookean spring of stiffness $k$, which we varied, and equilibrium length $l_{0}=1.12 \sigma$, exactly at the minimum of the Lennard Jones potential. The end atoms of the junction are connected each to one atom from the two NPs so that the equilibrium length of the junction is $L_{0}=(N+1) l_{0}$. However, in order to keep the junction locally perpendicular to the NP, second-neighbour interactions are added in the form of soft springs of stiffness $k=30 \epsilon / \sigma$ and equilibrium length $l_{0}^{\prime}=1.59 \sigma$. Apart from the elastic springs, the atoms of the junction interact with their neighbours through the bending energy term $\mathcal{H}=\kappa(1+\cos \theta)$, where $\theta$ is the angle between

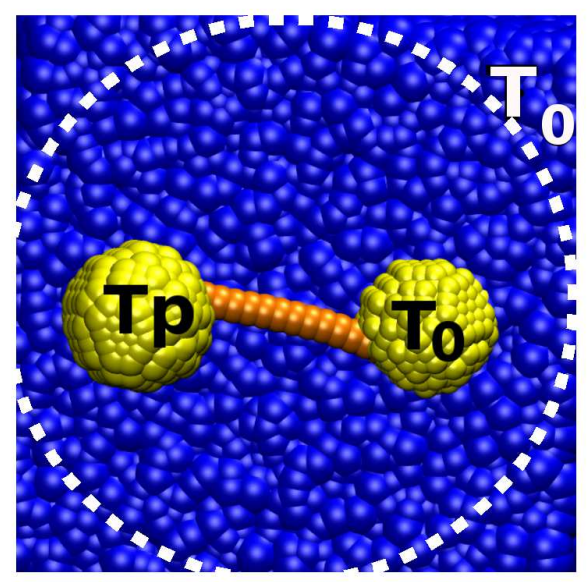

FIG. 1: Snapshot of a wet junction (with $N=13$ beads) between two NPs embedded in a Lennard Jones liquid. The region beyond the dashed line is thermostatted at $T_{0}$.

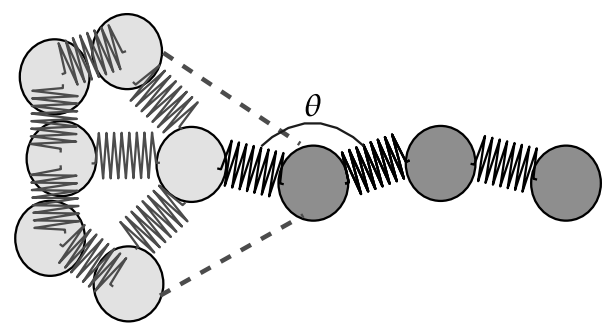

FIG. 2: Schematic representation of the junction. The dark circles represent the beads forming the junction, which interact via Hookean springs (black wavy lines); the light circles represent NP atoms and these interact through FENE springs. The soft springs between the NPs and the junction are indicated by dashed lines.

two successive segments, and $\kappa$ is the bending constant which sets the rigidity of the chain; in what follows, we set $\kappa=1000 \epsilon$ which yields a stiff molecular junction with a persistence length $l_{p} \simeq 2000 l_{0}$ much larger than the equilibrium length of the junction, even for the longest junctions considered $(N=25)$. Finally, each bead of the junction interacts with the atoms in the NPs through the Lennard-Jones potential $V_{\mathrm{LJ}}(r)=4 \epsilon\left((\sigma / r)^{12}-(\sigma / r)^{6}\right)$, where $\epsilon$ and $\sigma$ fix the units of energy and length, respectively, which we set to 1 , as we also do for the mass $m$ of all atoms. For wet junctions, the particles in the liquid interact via Lennard-Jones potentials with the same parameters $\epsilon$ and $\sigma$, as do the the atoms of both the junction and the NPs with the liquid. In what follows, all quantities are expressed in Lennard-Jones units (LJU), which are based on the elementary units $\epsilon, \sigma$ and $m$.

To prepare the wet junction in an equilibrium state, 


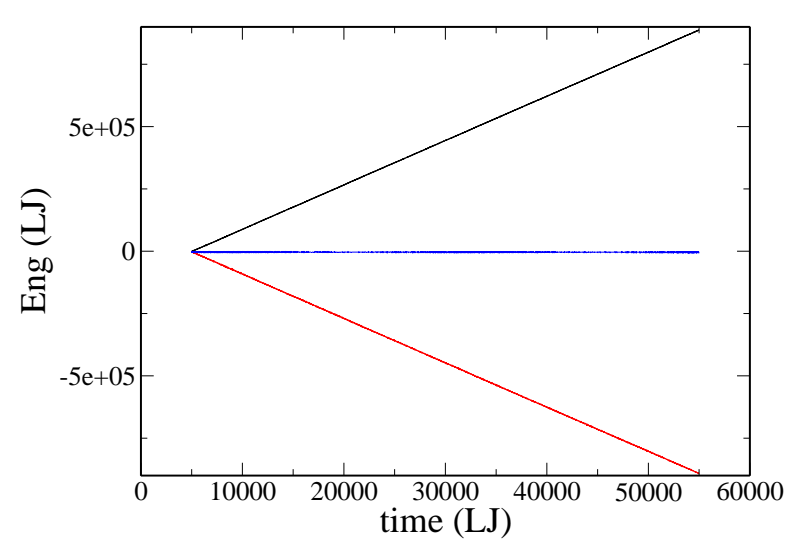

FIG. 3: Heat conduction across the dry, $N=5$ junction with $k=6000$ as a function of time: energy supplied to the hot NP (black line), energy removed from the cold NP (red line) and sum of the two (blue line).

the whole system is first equilibrated at temperature $T_{0}=0.75 \epsilon / k_{B}$ under pressure $P_{0}=0.0015 \epsilon / \sigma^{2}$ using a Nosé-Hoover thermostat. For the dry junction, we thermalize the junction and the NPs at the temperature $T_{0}$ and remove the total angular momentum of the system to avoid the possibility of a permanent rotational motion. To speed up equilibration in the latter case, we have applied a small friction force to all atoms in the system, running over 500,000 time steps $\left(d t=0.002 \sqrt{m \sigma^{2} / \epsilon}\right)$. After this initial equilibration period, for both wet and dry junctions, one of the two NPs is heated up to constant temperature $T_{p}>T_{0}$ using velocity rescaling while the temperature of the other NP is maintained at $T_{0}$. In presence of a liquid matrix, the region at distance $>10 \sigma$ from the surface of the NP, schematically represented in Fig. [1. is thermostatted at $T_{0}$; the whole system is also maintained at constant pressure $P_{0}$. Under these conditions, the energy transferred from one NP to the other across the junction corresponds to the amount of energy necessary to maintain the cold NP at $T_{0}$.

\section{CONDUCTIVITY OF DRY VERSUS WET JUNCTIONS}

Figure 3 displays the energy transferred across a short $(N=5)$ dry junction with $k=6000$ as a function of time. The spring stiffness is chosen here such that the characteristic pulsation (angular frequency), $\omega=\sqrt{k / m}$, matches the density of states (DOS) of the NPs, as will be discussed below. The junction is found to conduct energy; Fig. 3 shows that almost immediately after heating the junction, the energy supplied to the hot NP is exactly compensated by the energy removed from the cold NP, and the junction enters a steady state regime: the energy transferred from one NP to the other increases linearly with time, i.e., the power $P$ flowing through the junction

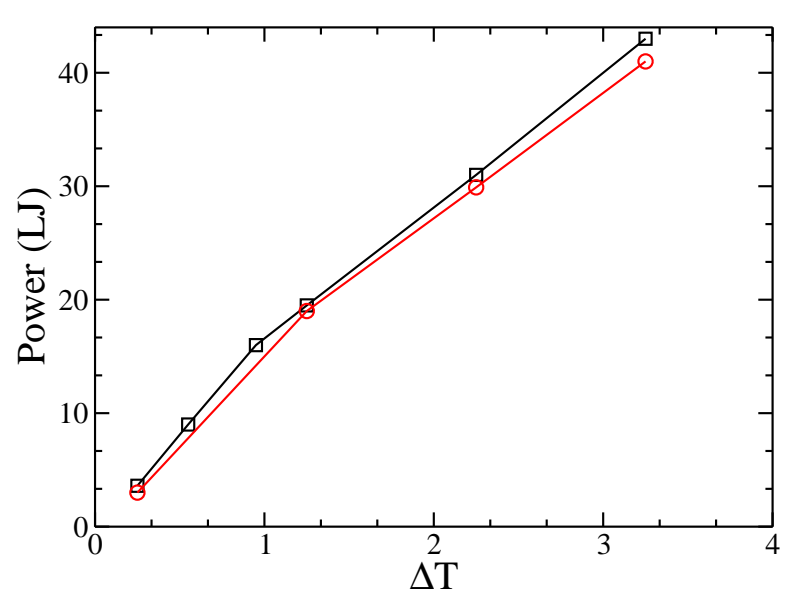

FIG. 4: Power as a function of the temperature difference $\Delta T$ for a short $(N=5)$ dry junction (o, black line) and wet junction ( $\square$, red line). The lines are guides to the eye.

is constant.

The corresponding results for the power as a function of the temperature difference $\Delta T$ between the hot and cold NP are presented in Fig. 4 . As expected, the power increases with $\Delta T$. A linear behaviour, is observed for temperature difference smaller than 1 LJU, which corresponds in real units to hundreds of Kelvins. This linear behaviour allows one to define unambiguously the conductance of the junction. With these temperature differences, the typical power is a few tenths of $\mathrm{nW}$. For larger $\Delta T$ 's, the power increases more slowly, a consequence of anharmonic contributions. Indeed, the atoms of the NP are connected with non linear springs which, at high temperature, may couple strongly with the springs from the junction, making the hole system strongly anharmonic. Fig. 4 shows also that the energy transfer is not affected by the presence of a liquid surrounding the system, at least for short junctions; this suggests that the heat flow via the liquid is negligible compared to that across the junction.

To verify this statement, we have run simulations without a junction, i.e. for two NPs at a surface-to-surface distance equal to the equilibrium length of the $N=5$ junction considered above, $L_{0}=6 l_{0} \simeq 7 \sigma$. In practice, this is achieved by attaching the center of mass of each NP to a fixed point using a stiff spring. The two NPs are immersed in the same liquid as the NP-junction system and equilibrated, after which one of the NP is heated up to temperature $T$ while the other is thermostatted to $T_{0}$ (and so is the liquid at distance $>10 \sigma$ ). In this situation, the liquid between the two NPs conducts energy due to the local temperature gradient (which is $\sim 1 \mathrm{~K} / \mathrm{nm}$ !). The conductance of the liquid is obtained by measuring the energy necessary to thermalize the cold NP.

Fig. 5 provides a comparison of the energy transferred across short wet junctions for different values of the spring constant $k$ to the energy transferred across the 


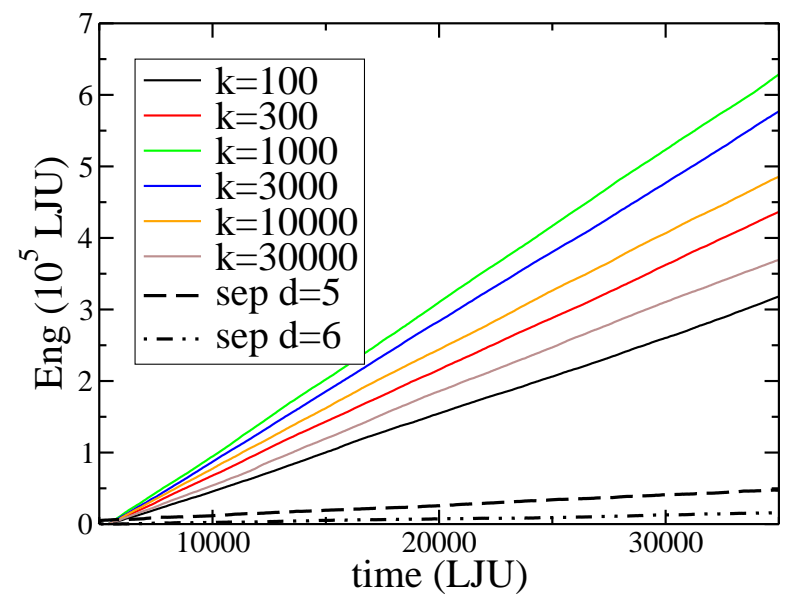

FIG. 5: Energy transferred across the wet junction compared to the energy transferred across the liquid, for a short $(N=5)$ junction and for a pure liquid with a comparable interparticle separation, respectively $d=5$ (dashed lines) and $d=6 \sigma$ (dashed-dotted lines). The temperature difference between the two contacts of the junction is $\Delta T=1.15$.

liquid alone. The range of values of $k$ has been chosen so as to cover all possible situations of overlap between the DOS of the NPs and the vibration spectrum of the junction. In particular, for the lowest value $(k=100)$, the characteristic pulsation $\omega=\sqrt{k / m}$ is smaller than the low frequency branch of the NP DOS, and one therefore expects weak energy transfer in this case. Indeed, the energy transferred across the liquid is negligibly small compared to the energy transferred across the junction. The power flowing across the $1 \mathrm{~nm}$ thick liquid layer is approximately $1 \mathrm{nW}$.

So far, we have considered a perfectly wetting (hydrophilic) situation. For large contact angles (i.e., a more hydrophobic particle) - which can be achieved by tuning the attractive part of the NP-liquid interaction - one expects even smaller energy transfer capability across the liquid. Indeed, at the interface between the NPs and the liquid, the temperature field displays a discontinuity due to the finite conductance of the interface 23]; this discontinuity is known to increase with hydrophobicity, as the mismatch between the two media decreases the transmission of phonons through the interface. For our two NPs and given the length $L$ of the junction, the effective temperature gradient in the liquid decreases as hydrophobicity increases because the temperature jump at the NP-liquid gets larger (i.e., the temperature of the liquid is smaller). Hence, the effective conductance of the liquid is expected to be even lower than in the hydrophilic case.

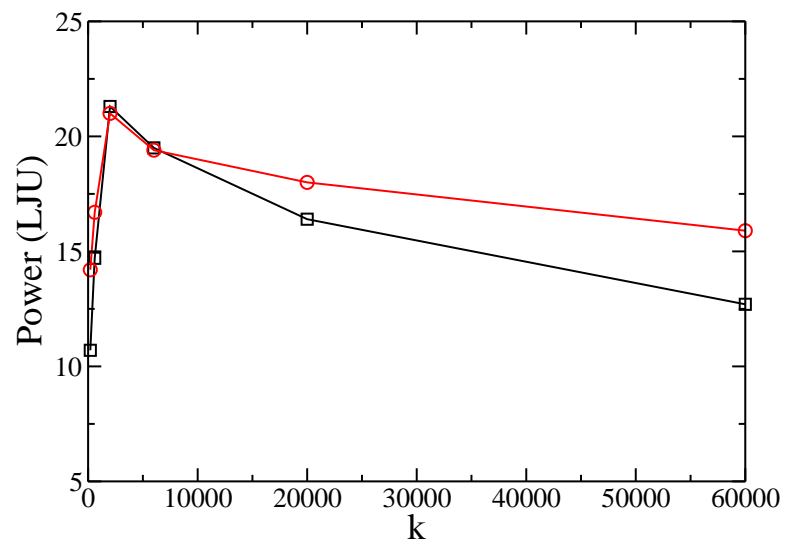

FIG. 6: Power as a function of spring stiffness $k$, for wet ( $\square$ ) and dry (o) short $(N=5)$ junctions; $\Delta T=1.15$.

\section{INFLUENCE OF THE STIFFNESS}

We now examine the variation of the conductance with the stiffness of the springs connecting the atoms within the junction. The results are presented in Fig. 6f for both the wet and the dry $N=5$ junction. The dependence on $k$ is found to be non monotonous in both cases: the power first increases, reaches a maximum - at the same value of $k(\simeq 2000)$ for both wet and dry junctions - , then decreases, the dry junction thus appearing to be a better conductor than the wet junction for a large stiffness.

This particular behaviour can be explained qualitatively in terms of the DOS of the NPs and the vibrational spectrum of the junction, as depicted in Fig. 7. The DOS of the NPs has been obtained by Fourier transforming the velocity autocorrelation function. For the junction, we have only considered, for the sake of clarity, the spectrum of stretching modes, whose resonance frequencies are given by $\omega_{p}=\sqrt{k / m} \sin (\pi p / 2 N), p$ being an integer between 1 and $N-1$. Apart for these $N-1$ modes, there are $N-2$ resonance frequencies corresponding to bending which, in the harmonic approximation, are decoupled from longitudinal modes so that the corresponding spectrum does not depend on $k$. Different cases may be encountered, depending on $k$, as demonstrated in Fig. 7. For small values of $k$, only a few modes of the junction overlap with the low frequency branch of the NP DOS, so that only the low frequency modes of the NP are excited and a small conductance is expected, as indeed observed in Fig. 6. When $k \simeq 2000$, the matching between the stretching modes and the NP DOS is optimal, and the junction is a very good conductor, consistent with the results of Fig. 6. For large values of $k$, the normal modes of the junction have high frequencies and there is little overlap with the NP DOS so that the energy transfer across the junction is expected to decline.

It turns out, however, that the conductance of stiff junctions is larger than that of soft junctions, either dry 


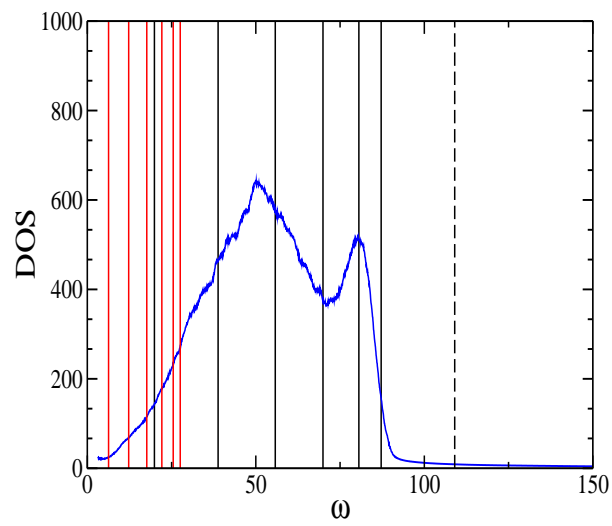

FIG. 7: Vibrational spectrum of a $N=5$ junction (vertical lines) superimposed on the NP DOS (full line) for different values of the longitudinal stiffness $k: k=200$ (red lines), 2000 (black lines) and 60000 (dashed line in this case, only the lowest frequency mode is shown).

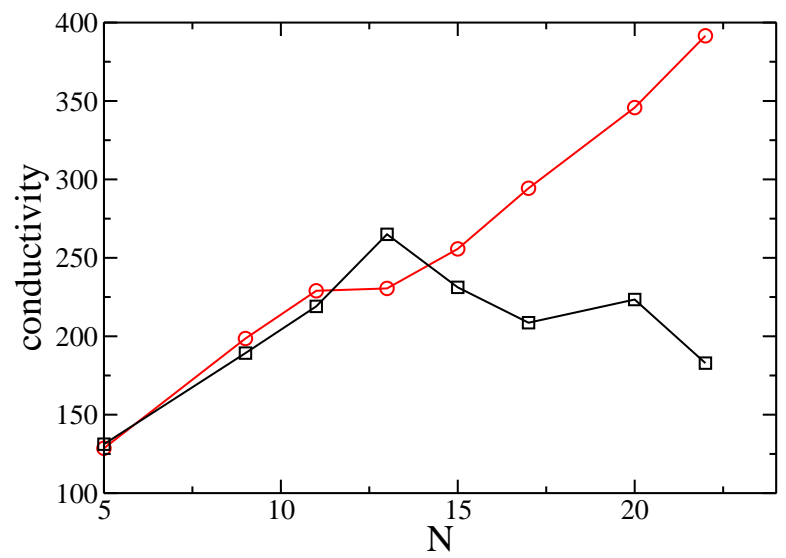

FIG. 8: Conductivity of dry (०) and wet ( $\square$ )junctions as a function of length, measured by the number of beads of the junction $N$. Here, $k=6000$ and $\Delta T=1.15$.

or wet (cf. Fig. 6). This may appear counterintuitive, as there is some overlap between the soft-junction spectrum and the NP DOS while there is none for the stiff junction. A possible explanation is that, for large stiffness, the high-frequency longitudinal modes of the junction excite higher harmonics of the NPs, allowing energy to be transferred between them. As noted earlier, Fig. 6 also reveals that the dry junction conducts heat better than the wet junction. The difference may be understood in terms of the friction of the liquid, which damps the low frequency modes of the NPs, thus reducing the efficiency of the non-linear coupling discussed above.

\section{EFFECT OF LENGTH}

We discuss, finally, how the length of the junction affects the energy transfer between the NPs. The variation of the conductivity of dry and wet junctions with length is displayed in Fig. 8. The stiffness $k(=6000)$ has been chosen here so as to ensure a good matching between the junction and the thermal contacts with the NPs. For the dry junction, the relation between conductivity and length is monotonous: the conductivity increases almost linearly, i.e., the conductance is approximately constant. This behaviour is standard for unidimensional systems, and easy to understand. Upon increasing the length of the junction, the number of vibrational modes $N_{\text {modes }}=2 N-3$ increases proportionally and the overlap with the NP DOS increases, provided the normal mode frequencies $\omega_{\text {stretching }}=\sqrt{k / m}$ and $\omega_{\text {bending }}=\sqrt{\kappa / l_{0}^{2}}$ are not too small. This is demonstrated in Fig. 9 where we superimpose the spectra of free dry junctions (not connected to nanoparticles) of two different lengths $(N=5$ and $N=20$ ) with the NP DOS: the overlap clearly increases with length and, as a result, the conductivity increases.

For wet junctions, the scenario is different, as can be seen in Fig. 8. The conductivity first increases with length, following closely the results for the dry junction; for $N>13$, however, the current starts decreasing with increasing length, i.e. the conductance drops. This non monotonous behaviour may be understood by analysing the vibrational spectra of wet junctions, also shown in Fig. 9. These have been calculated from simulations of a free junction (i.e., without NPs) immersed in a liquid. For the shortest junction $(N=5)$, the DOS of wet and dry junctions are very similar, except perhaps at low frequencies; this is evidently consistent with the fact that the conductivities of short junction is not affected by the presence of the surrounding solvent, as observed above (Fig. 8). Fig. 9 however shows that the spectra of long wet and dry junctions may be different and, in particular, the low-frequency modes of the dry junction are absent in the wet junction. We infer from this observation that the surrounding solvent damps the low-frequency modes of the wet junction. To ascertain this, we also show in Fig. 9 the DOS of the Lennard-Jones liquid, here thermostatted at the reference temperature $T_{0}=0.75$. It is found to be significant only at low frequencies - $\omega$ less than 40 or so; in contrast, for higher frequencies, the spectrum of the liquid is vanishingly small. Hence, highfrequency modes of the junction feel the presence of the solvent as a frictionless elastic medium, and are thus not affected by the presence of the liquid. This explains why the high-frequency part of the spectrum of dry and wet junctions are very similar, irrespective of the length of the junction. Low-frequency modes, on the other hand, feel the solvent as a viscous medium, and are therefore significantly damped by the solvent. This is can in fact be seen in Fig. 9 where the low-frequency modes are almost completely absent, more precisely almost completely damped - in the spectrum of the long wet junction. 


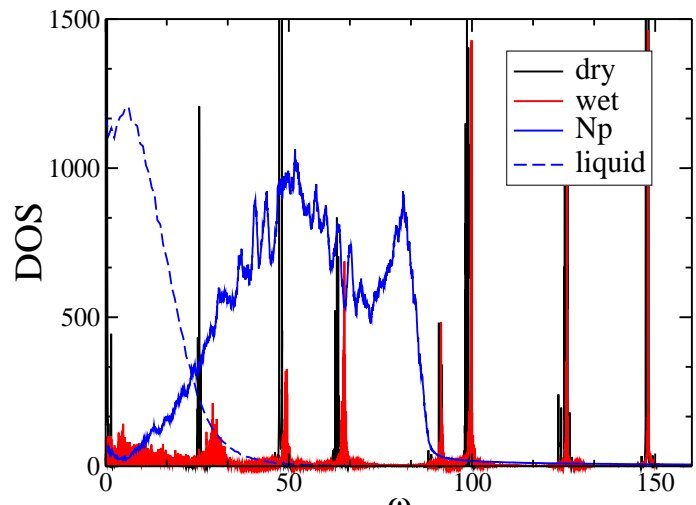

$\omega$

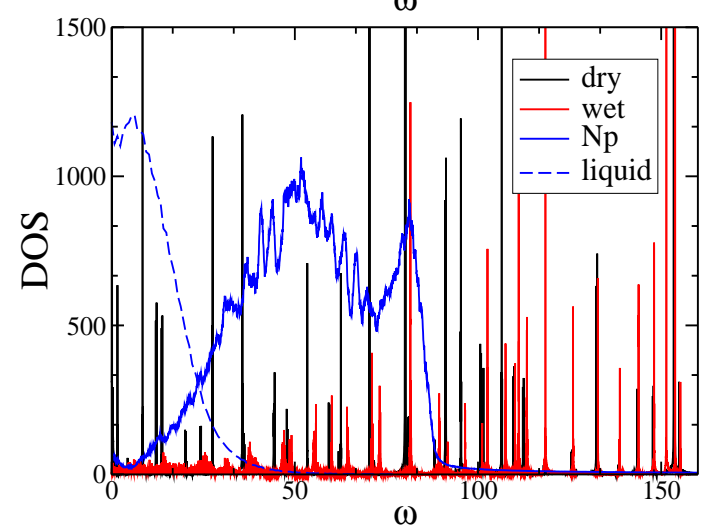

FIG. 9: Vibrational spectra of dry (black lines) and wet (red lines) junctions with $N=5$ (top) and $N=20$ (bottom), superimposed on the DOS for the NPs (blue solid lines) and that of the liquid (dashed blue lines).

\section{CONCLUSION}

We have studied the problem of heat transfer accross a molecular junction between two nanoparticles that lie either in vacuum and in a liquid environment using classical molecular dynamics simulations. While our model remains "generic", it does capture the essential physics of the problem. For the junction in vacuum, the well-known result of a conductance that is independent of length is recovered; however, the conductivity depends sensitively on the overlap between the density of states of the junction itself and that of the nanoparticles to which it is connected. The presence of a liquid is found to affect this behaviour in a significant manner: the conductance is constant to a crossover length, above which it starts to decline and the standard Fourier regime is recovered. Our results can be rationalized in terms of the overlap between the vibrational spectrum of the junction and that of the immersing liquid, which is qualitatively different in short and long junctions.
We acknowledge funding from the Agence Nationale de la Recherche (ANR) project Opthermal, as well as the Natural Sciences and Engineering Research Council of Canada (NSERC) and the Fonds Québécois de la Recherche sur la Nature et les Technologies (FQRNT). Part of the simulations have been done at the Pôle Scientifique de Modélisation Numérique (Lyon) using the LAMMPS package freely available at http://lammps.sandia.gov - see 24]

* Electronic address: samy.merabia@univ-lyon1.fr

† Electronic address: jean-louis.barrat@ujf-grenoble.fr

‡ Electronic address: laurent.lewis@umontreal.ca

[1] E. Fermi, J. Pasta and S. Ulam, Los Alamos report LA1940, 1955.

[2] D.G. Cahill, W.K. Ford, K.E. Goodson, G.D. Mahan, A. Majumdar, H.J. Maris, R. Merlin, S.R. Philpot, J. App. Phys. 200393 (2) 793.

[3] A. Dhar, Adv. Phys. 57, 457-537, 2008.

[4] C.W. Chang, D. Okawa, A. Majumdar and A. Zettl, Science 20063141121.

[5] G. Casati, Nature nanotechnology, 2007223.

[6] A. Dhar and D. Roy, Jour. Stat. Phys. 2006125805.

[7] D. Segal, A. Nitzan and P. Hnggi, J. Chem. Phys. 2003 119 (13), 6840.

[8] Y. Zhou and D. Segal, J. Chem. Phys. 2010133094101.

[9] N. Mingo and D.A. Broido, Phys. Rev. Lett. 200595 096105.

[10] T. Luo and J.R. Lloyd, Int. J. Heat Mass. Transf. 2010 531.

[11] N. Mingo, Phys. Rev. B 200674125402.

[12] A. Henry and G. Chen, Phys. Rev. Lett. 2008101235502.

[13] T.S Tighe, J.M. Worlock, M.L. Roukes, App.Phys. Lett. 1997 70, 2687.

[14] K. Schwab, E.A. Henriksen, J.M. Worlock and M.L. Roukes, Nature 2000404974.

[15] S. Berber, Y.K. Kwon and D. Tomanek, Phys. Rev. Lett. 2000844613.

[16] P. Kim, L. Shi, A. Majumdar, P.L. McEuen, Phys. Rev. Lett. 200187215502.

[17] C. Yu, L. Shi, Z. Yao, D. Li, A. Majumdar, NanoLett. 200551842.

[18] C.W. Padgett, O. Shenderova and D.W Brenner, NanoLett. 200661827.

[19] R. Landauer, Philosophical Magazine 197021 (172) 863.

[20] S. Merabia, P. Keblinski, L. Joly, L. Lewis and J.-L. Barrat, Phys. Rev. E 200979021404.

[21] S. Merabia, S. Shenoguin, L. Joly, P. Keblinski and J.-L. Barrat, PNAS 200910615113.

[22] K. Kremer and G.S. Grest, J. Chem. Phys. 1990925057.

[23] J.-L. Barrat and F. Chiaruttini, Mol. Phys. 2003101 1605.

[24] S. Plimpton, J. Comp. Phys., 1995 117, 1-19. 